\title{
Post-Traumatic Epilepsy Associations with Mental Health Outcomes in the First Two Years after Moderate to Severe TBI: A TBI Model Systems Analysis.
}

Shannon B. Juengst ${ }^{\mathrm{a}, 1}$, Amy K Wagner ${ }^{\mathrm{a}-\mathrm{e}}$, Anne C Ritter ${ }^{\mathrm{a}, \mathrm{b}}$, Jerzy P Szaflarski ${ }^{\mathrm{f}}$, William C Walker $^{\mathrm{g}}$,Ross D Zafonte ${ }^{\mathrm{h}}$, Allen W Brown ${ }^{\mathrm{i}}$, Flora M Hammond ${ }^{\mathrm{j}, \mathrm{k}}$, Mary Jo Pugh, ${ }^{\mathrm{l}, \mathrm{m}}$, Timothy Shea $^{\mathrm{n}}$, Jason W Krellman ${ }^{0}$, Tamara Bushnik ${ }^{\mathrm{p}}$, Patricia M Arenth ${ }^{\mathrm{a}}$

${ }^{\text {a }}$ Physical Medicine \& Rehabilitation, University of Pittsburgh, Pittsburgh, PA, United States

${ }^{\mathrm{b}}$ Department of Epidemiology, University of Pittsburgh, Pittsburgh, PA, United States

c Safar Center for Resuscitation Research, University of Pittsburgh, Pittsburgh, PA, United States

${ }^{\mathrm{d}}$ Department of Neuroscience, University of Pittsburgh, Pittsburgh, PA, United States

${ }^{\mathrm{e}}$ Center for Neuroscience at University of Pittsburgh, Pittsburgh, PA, United States

${ }^{\mathrm{f}}$ University of Alabama at Birmingham Department of Neurology and UAB Epilepsy Center, Birmingham, AL, United States

${ }^{g}$ Dept of Physical Medicine \& Rehabilitation, Virginia Commonwealth University, Richmond, VA, United States

h Spaulding Rehabilitation Hospital, Massachusetts General Hospital, Brigham and Women’s Hospital, Harvard Medical School, Boston, MA, United States

${ }^{\mathrm{i}}$ Department of Physical Medicine and Rehabilitation, Mayo Clinic, Rochester, MN, United States

${ }^{j}$ Carolinas Rehabilitation, Charlotte, NC, United States

${ }^{\mathrm{k}}$ Indiana University School of Medicine, Indianapolis, IN, United States

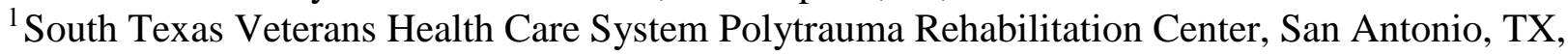
United States

${ }^{\mathrm{m}}$ Department of Epidemiology and Biostatistics, University of Texas Health Science Center San Antonio, San Antonio, TX, United States

${ }^{\mathrm{n}}$ Department of Physical Medicine and Rehabilitation, Ohio State University, Columbus, $\mathrm{OH}$, United States

${ }^{\circ}$ Department of Neurology, Columbia University Medical Center, New York, NY, United States

${ }^{\mathrm{p}}$ Rusk Rehabilitation, New York University School of Medicine, New York, NY, United States ${ }^{1}$

\section{Corresponding Author: $\quad$ Amy K. Wagner, MD}

Kaufmann Medical Building, Ste 202

3471 Fifth Ave.

Pittsburgh, PA 15213

Phone: 412-648-6666 Fax: 412-692-4354

wagnerak@upmc.edu

\footnotetext{
${ }^{1}$ Department of Physical Medicine \& Rehabilitation, Department of Rehabilitation

Counseling, University of Texas Southwestern Medical Center, Dallas, TX, United States
}

This is the author's manuscript of the article published in final edited form as: 
PTE and mental health after TBI

\begin{abstract}
Purpose: Research suggests there are reciprocal relationships between mental health (MH) disorders and epilepsy risk. However, $\mathrm{MH}$ relationships to post-traumatic epilepsy (PTE) have not been explored. Thus, the objective of this study was to assess associations between PTE and frequency of depression and/or anxiety in a cohort of individuals with moderate-to-severe TBI who received acute inpatient rehabilitation.
\end{abstract}

Methods: Multivariate regression models were developed using a recent (2010-2012) cohort ( $\mathrm{n}=867$ unique participants) from the TBI Model Systems (TBIMS) National Database, a time frame during which self-reported seizures, depression [Patient Health Questionnaire (PHQ)-9], and anxiety [Generalized Anxiety Disorder (GAD-7)] follow-up measures were concurrently collected at year-1 and year-2 after injury.

Results: PTE did not significantly contribute to depression status in either the year-1 or year-2 cohort, nor did it contribute significantly to anxiety status in the year-1 cohort, after controlling for other known depression and anxiety predictors. However, those with PTE in year-2 had 3.34 times the odds ( $p=.002)$ of having clinically significant anxiety, even after accounting for other relevant predictors. In this model, participants who self-identified as Black were also more likely to report clinical symptoms of anxiety than those who identified as White. PTE was the only significant predictor of comorbid depression and anxiety at year-2 (Odds Ratio $2.71 ; \mathrm{p}=0.049)$

Conclusions: Our data suggest that PTE is associated with MH outcomes 2 years after TBI, findings whose significance may reflect reciprocal, biological, psychological, and/or experiential factors contributing to and resulting from both PTE and MH status post-TBI. Future work 
PTE and mental health after TBI

should consider temporal and reciprocal relationships between PTE and MH as well as if/how treatment of each condition influences biosusceptibility to the other condition.

Keywords: traumatic brain injury, post-traumatic epilepsy, mental health disorders, depression, anxiety, traumatic brain injury model system 
PTE and mental health after TBI

\section{Introduction}

Post-traumatic seizures (PTS), and the development of epilepsy (PTE), are common after traumatic brain injury (TBI) and contribute substantially to the medical and comorbid health burden that individuals often have after TBI.[1] PTS are classified with reference to time postinjury as immediate (<24hrs), early (24hrs-7d), or late ( $>7 \mathrm{~d}$ post-TBI),[2] reflecting proposed differences in causal mechanisms and subsequent seizure risk.[3] While immediate and early PTS are likely directly related to the primary injury, late PTS (the definition of PTE) are likely due to ongoing secondary injury cascades that contribute to epileptogenesis.[4] Though PTE prevalence varies widely based on population characteristics, including injury severity, a recent study using the TBI Model System National Database (TBIMS-NDB), composed of individuals with moderate-to-severe TBI, reported that 1.8\% had incident PTS between 7 days post-injury and inpatient discharge, 9.2\% developed PTE between inpatient discharge and follow-up at year1 post-injury, and 5\% developed PTE between year-1 and year-2 post-injury, with a cumulative PTE incidence of $16.8 \%$ by 2 years post-TBI [5].

The high risk for sudden death and decreased life expectancy among those with epilepsy compared to the general population are due both to seizure-related and external causes, including suicide and fatal accidents.[6] After TBI, individuals have 33-50 times higher likelihood of unexpected mortality due to seizures compared to the general population.[7] Health-related quality of life is often poor among individuals living with epilepsy, in part due to comorbid disease burden.[8] To date, there is scant literature evaluating the effects of PTE, beyond mortality, after TBI. One small study involving 91 subjects enrolled in the TBIMS suggests individuals with PTE are more dependent with their transportation needs, have more functional 
PTE and mental health after TBI

disability, and report lower life satisfaction up to 5 years post-injury compared to similar individuals without PTE.[9]

The most common comorbid conditions associated with epilepsy in the general population are mood disorders, followed closely by anxiety [10]. Multiple reports in the literature suggest a high rate of comorbid psychiatric disorders across the age span among individuals living with epilepsy. Among children with epilepsy, rates as high as $35-50 \%$ have been documented.[11] In adults with epilepsy and self-reported employment difficulties, 67\% had psychiatric comorbidities.[12] Another small cohort of individuals with epilepsy referred for neuropsychiatric services reported that $38.3 \%$ of the population had concurrent depression and 26.7\% had concurrent anxiety.[13] A population-based study demonstrated that individuals with epilepsy have an adjusted odds ratio of 5.44 for psychiatric/neurodevelopmental comorbidities vs. the general population.[14]

Regarding prevalence of psychiatric conditions after TBI, a seminal study found over $50 \%$ of individuals with TBI experienced depression at some point in the first year after injury, with point prevalence as high as 31\%.[15] Other studies using TBIMS-NDB data found that 26\% of participants reported symptoms of major depressive disorder at year-1 post-injury with over 50\% of these participants still reporting depression at year-2.[16,17] An additional 22\% reported symptoms of minor depression at year-1,[16] of whom one-third went on to report major depression at year-2 after injury.[17] Depression remained common (24.8-28.1\%) up through 20 years post-TBI and was associated with a high risk for suicidal behavior.[18] Risk factors for depression after TBI include younger age, pre-injury mental health (MH) treatment, substance abuse, functional disability, and being female.[16] Further, there is a high degree (60\%) of comorbid anxiety.[15] Approximately 21\% of individuals with TBI report clinically significant 
anxiety a year after injury, with similar risk factors as depression, including middle age, black race, lower socioeconomic status, pre-injury MH treatment, and previous TBI.[19]

No study to date has examined the association between PTE and MH outcomes after TBI, despite the documented effect of epilepsy on MH in the general population with epilepsy. While our recent study showed that history of MH treatment prior to TBI was a significant risk factor for PTE during the first year post-injury,[20] no studies have examined the effects of PTE on concurrent or subsequent $\mathrm{MH}$ outcomes. Therefore, the aims of this study were to examine the differences in MH (depression and anxiety) by PTE status at 1 and 2 years after TBI among individuals enrolled in the TBIMS-NDB by building on previous TBIMS-NDB predictive models for post-TBI depression[16] and anxiety,[19] and to determine whether PTE is an independent risk factor for MH outcomes after TBI.

\section{Methods}

\subsection{Participants}

This study is a secondary analysis of the prospective, longitudinal TBIMS-NDB. Inclusion criteria for the TBIMS-NDB are: 1) a documented moderate/severe TBI, defined as any one of the following: post-traumatic amnesia $>24$ hours, loss of consciousness $>30$ minutes, emergency department Glasgow Coma Scale score <13, or positive neuroimaging; 2) $\geq 16$ years old; 3) admitted to a participating hospital within 72 hours of injury with ongoing acute care and inpatient rehabilitation at a TBIMS hospital. Written informed consent was obtained, and details about enrollment and data collection in the TBIMS-NDB are available here: https://www.tbindsc.org/Syllabus.aspx 
Individuals enrolled in the TBIMS-NDB received acute care at an affiliated trauma center and received acute inpatient rehabilitation, for which individuals are determined to have capacity to participate in and benefit from a multidisciplinary therapy program. For this study, participants were restricted to those who had a TBI between July 2010 and November 2012, based on having a 1 or 2 year follow-up during the time frame in which seizure, depression, and anxiety follow-up measures were concurrently collected. The seizure variable was added to the TBIMS-NDB in October of 2012. The depression and anxiety variables were removed from the database in October of 2013. Further, this sample was restricted to those who were capable of providing self-report at follow-up, as the mental health measures require self-report. Capacity for self-report was determined by trained TBI-MS assessors following a standard protocol. A breakdown of included participants is provided in Figure 1. There were 867 unique participants, with 453 participants in the year 1 cohort and 434 in the year 2 cohort; due to restrictions in data collection noted above, only 20 participants were in both cohorts, so longitudinal data were not available.

\subsection{Measures}

\subsubsection{Demographic and Clinical Covariates.}

Age, sex, race, and inpatient rehabilitation discharge Functional Independence Measure $^{\mathrm{TM}}$ (FIM) were collected through medical chart abstraction, and FIM at follow-up was collected via participant or family member interview. Age was further categorized into young, middle-age, and older adults (16-30, 31-60, and 61+ years), to allow for nonlinear relationships based on previous findings of nonlinear relationships between age and psychological variables (depression, anxiety) in TBI $[19,21]$. Post-traumatic amnesia (PTA) served as an indicator of 
PTE and mental health after TBI

initial injury severity, as it has been found to be a better predictor of outcome than other acute brain injury severity measures.[22] Pre-injury MH treatment (ever treated for mental health problem), previous TBI, and substance abuse (calculated based on self-reported alcohol and/or drug use) at time of follow-up were collected via medical chart abstraction or participant/proxy interview. Data on acute seizures were derived from International Classification of Diseases (ICD9) codes from the acute inpatient hospital stay; acute seizures included any seizure that occurred during the acute-care hospitalization and was assigned an ICD9 code, regardless of time since injury (e.g. potentially including immediate, early, and late seizures).

\subsubsection{Post-traumatic Epilepsy.}

PTE status at each time point was collected via participant report, with data collection beginning for all follow-up visits in the TBIMS-NDB in October of 2012. Specifically, participants were asked a yes/no question about having had a seizure since discharge from rehabilitation (year-1) or in the past year (year-2). This means that for the year-2 cohort, PTE referred only to seizures that had occurred between year- 1 and year-2 post-injury. Given the time frame during which these seizure data were collected, information on whether participants in the year- 2 cohort had seizures between acute care discharge and year- 1 post-TBI was not available. Therefore, PTE reported in this study reflects in-year prevalence and not incidence of PTE. Though potentially limited by poor recall and recall bias, self-report is still the most feasible method for data collection in large epidemiologic studies and is a common practice in studies on seizures/epilepsy.[23] A study of test-retest reliability conducted on TBIMS-NDB outcomes measures found perfect test-retest reliability (kappa=1.00) for self-reported seizures in the last year. [24]

\subsubsection{Depressive symptoms.}


Depressive symptoms at year-1 and year-2 were measured with the Patient Health Questionnaire 9 (PHQ9), a self-reported measure of depressive symptoms rating 9 hallmark symptoms of depression on a 0-3 point scale, which has been validated for use after TBI.[25] Depressive symptom severity was measured by PHQ9 total scores (range 0-27). Depression status was categorized based on PHQ9 scores as depressed $(\geq 10)$ or not depressed $(<10)$, which has demonstrated a sensitivity of $88 \%$ and specificity of $90 \%$ for identifying clinical depression after TBI.[25] PHQ9 data collection was discontinued in the TBIMS-NDB in October of 2013.

\subsubsection{Anxiety symptoms.}

Anxiety symptoms at year-1 and year-2 were measured with the General Anxiety Disorder 7 (GAD7), a self-reported measure of anxiety symptoms rating 7 symptoms of general anxiety on a 0-3 point scale.[26] Anxiety symptom severity was measured with GAD7 total scores (range 0-21), and clinically significant anxiety (anxiety status) was categorized as present ( $\geq 8$ ) or absent $(<8)$, based on previously established thresholds sensitive to anxiety.[19,26] GAD7 data collection was discontinued in the TBIMS-NDB in October of 2013.

\subsection{Data Analysis}

Data were analyzed separately in each cohort (year-1 and year-2) using SPSS v24 for Windows. Group differences in anxiety and depression were analyzed comparing those with and without PTE using Mann-Whitney U tests based on data distribution. We then tested the unique contribution of PTE to depression status and anxiety status using logistic regression. Covariates in the base models for depression included age, sex, race, pre-injury MH treatment, inpatient rehabilitation discharge FIM scores, and concurrent substance abuse, based on a prior depression 
study in the TBIMS.[16] Covariates in the base models for anxiety included age, sex, race, and pre-injury MH treatment, based on a previous anxiety study using the TBIMS cohort.[19] Previous TBI was excluded due to a very small number $(n=9)$ of participants reporting it. The alpha level for statistical significance was set at .05 for two-tailed tests.

\section{Results}

Demographic and clinical characteristics of participants in both the year-1 $(n=453)$ and year-2 ( $\mathrm{n}=434)$ cohorts by PTE status are presented in Table 1. Comparing the two cohorts, a larger percentage of participants in the year- 1 cohort had PTE $\left(\chi^{2}=4.67, \mathrm{p}=0.032\right)$ and reported $\mathrm{MH}$ treatment prior to injury $\left(\chi^{2}=5.54, \mathrm{p}=0.020\right)$ compared to the year- 2 cohort. However, a significantly larger percentage of participants in the year- 2 cohort had an acute seizure $\left(\chi^{2}=9.79\right.$, $\mathrm{p}=0.002$ ) during their inpatient hospitalization stay compared to the year-1 cohort. There were no significant differences between cohorts with regard to age, sex, race, previous TBI, PTA, FIM at inpatient rehabilitation discharge, FIM at follow-up, substance abuse, depression (symptoms or status), or anxiety (symptoms or status). Among those with in-year seizures, 13 (24.1\%) reported being hospitalized at least once for seizures in year-1 and 12 (36.4\%) reported being hospitalized at least once for their seizures in year-2.

Depression and anxiety symptoms, status, and comorbidity by PTE status in each cohort are presented in Table 2. All MH measures differed significantly $(\mathrm{p}<.01)$ by PTE status in the year-2 cohort, and while there were no statistically significant group differences in the year-1 cohort, values were nominally in the same direction as in year-2. Specifically, those with PTE had a higher frequency of both depression and anxiety and reported more depressive and anxiety 
symptoms than participants without PTE. Nearly all participants with PTE in year-2 who reported anxiety also reported co-occurring depression.

In the logistic regressions, PTE did not significantly contribute to depression status in either the year- 1 or year- 2 cohort, nor did it contribute significantly to anxiety status in the year1 cohort, after controlling for other known predictors of depression and anxiety (data not shown). In the year-2 cohort, those with PTE in year-2 had 3.34 times the odds ( $\mathrm{p}=.002)$ of having clinically significant anxiety (Table 3), even after accounting for other relevant predictors. Participants who identified as Black were also more likely to report clinical symptoms of anxiety than those who identified as White. PTE was the only significant predictor of comorbid depression and anxiety at year 2 (Table 4).

\section{Discussion}

The existing research indicates bidirectional relationships between psychiatric conditions and epilepsy.[10,27] However, previous studies have not examined the influence of PTE on psychiatric conditions post-TBI. This could be partly due to the fact that PTE only makes up 5\% of the total incidence of epilepsy in population-based study;[28] however, it is one of the most common and serious long-term consequences of TBI [5]. We found no statistically significant associations between PTE and either depression or anxiety at year-1 after TBI, but at year-2 postTBI, both depression and anxiety symptoms differed by PTE status. PTE was predictive of anxiety and comorbid anxiety and depression at year-2 post-TBI, after controlling for other known predictors of MH outcomes post-injury. 
Whether the differences in the relationships between PTE and both depression and anxiety after TBI are due to temporal effects post-injury or cohort effects is unclear in the present study. The year-2 cohort had a lower percentage of participants reporting pre-injury $\mathrm{MH}$ treatment, a known predictor of post-injury psychiatric conditions.[16,19] Despite this, the year2 cohort had a much higher prevalence of both depression and anxiety than the year-1 cohort. The prevalence for depression and anxiety in the year-2 cohort (42.4\% and $42.2 \%)$ was similar to or higher than prevalence reported among those in the general population with epilepsy (38.3\% and 26.7\%).[13] This indicates that PTE has higher associations with new onset depression or anxiety post-TBI when compared to those with a pre-injury history of psychiatric conditions who may develop post-injury depression or anxiety regardless of PTE status (particularly in the first year post-injury). These new psychiatric conditions post-injury may develop as a result of shared biological pathology, psychological adjustment, or psychological trauma from experiencing seizures and their associated events. In both cohorts, there was a higher percentage of participants with pre-injury MH treatments among those with PTE compared to those without, which, while not statistically significant, is consistent with previous findings in both TBI and the general population with epilepsy that psychiatric conditions contribute to seizure risk.[10,20]

The larger percentage of participants in the year-1 cohort reporting pre-injury $\mathrm{MH}$ treatment may also indicate that more participants in this cohort were taking medication for $\mathrm{MH}$ disorders post-injury as well. Although medications were not tracked as a part of this study, this factor could explain the lower prevalence of both depression and anxiety in this cohort, if medications were effective for managing participants’ psychiatric conditions. MH treatment considerations are particularly salient, given that previous studies suggest individuals with TBI are at risk for physician-prescribed polypharmacy of psychotropic medications during their 
recovery course.[29] In fact, many psychotropic medications prescribed after TBI, including antipsychotics (25\% of those in inpatient rehabilitation programs) and antidepressants (67\% of those in inpatient rehabilitation programs), coupled with the frequent prescription of multiple psychotropic medications simultaneously (31.8\% receive $\geq 6$ psychotropic medications while participating in inpatient rehabilitation programs),[29] may contribute to seizure risk after TBI.[30] Medications for MH disorders, including some typical and atypical antipsychotics (e.g. clozapine) and dopaminergic stimulants (e.g. amantadine) can decrease seizure threshold or increase seizure risk.[16,31] A recent literature review suggests that tricylic antidepressants are associated with reduced seizure risk in a general population participating in antidepressant trials.[32] However, other antidepressant trials in the general population among individuals over the age of 65 (an age group with relatively high TBI risk), suggests some selective serotonin reuptake inhibitors (SSRIs) may increase seizure susceptibility,[33] despite experimental epilepsy studies indicating that high doses of some SSRIs may actually reduce seizure susceptibility.[34] Importantly, non-pharmacological approaches to MH treatment after TBI can be effective treatments and may help reduce occurrence of polypharmacy, and perhaps also seizure risk, after TBI.[35,36]

It is also possible that the differences across cohorts in PTE and MH relationships are due to latent temporal effects - biological, psychological, or environmental - that differ from 1 to 2 years post-injury and not captured in this study. While PTE was more prevalent in the year-1 cohort than in the year-2 cohort, a higher percentage of participants in the year-2 cohort had experienced acute (in-hospital) seizures, an established predictor of later seizures.[20] Additionally, more participants were hospitalized for their seizures in the year-2 cohort, suggesting that chronicity and/or severity of PTE may also have been an unmeasured factor in 
the relationship between PTE and MH outcomes. In contrast, a large epidemiological study also conducted using TBIMS data reported a cumulative incidence of PTE by 1 year post-TBI of 11.9\% and by 2 years post-TBI of 16.8\% (an additional 5\% experiencing incident seizures), but only 5.5\% of participants reported any seizure between years 1 and 2.[5] Therefore, though we cannot say how many participants in our year 2 cohort had incident seizures based on the available data, the number maybe similar to the number reporting any seizure in year 2 based on a larger study using comparable data.

In the first year post-TBI, individuals may still be actively participating in rehabilitation or more intensively involved in medical care to address various physiological consequences of injury. They may not yet be ready to return to various life roles that could be impacted by seizures, such as sports, school, or work. They may also develop incident depression or anxiety or engage in other risk behaviors (e.g. alcohol abuse), which could increase their risk for future seizures (e.g. in year 2). Given that depression does not often resolve, and that even minor depression frequently evolves into major depression in the second year after injury,[17] this known relationship could explain the stronger association between MH conditions and PTE in year-2. In the second year post-TBI, individuals may have returned to work, driving, or other meaningful activities, and the occurrence of PTS at this point could be more disruptive to this participation (as evidenced by the higher number of rehospitalizations noted in the year-2 cohort), resulting in poorer $\mathrm{MH}$ as individuals adjust to new disability. First-time seizures in year-2, which, as noted previously, likely make up the majority of seizures reported in year 2, could be regarded as unexpected, which may induce anxiety and/or hopelessness if individuals were otherwise having good recovery trajectories. 
Reciprocal associations between MH disorders and epilepsy have been demonstrated clinically in the general population with epilepsy,[27] and some studies show shared neural networks that may contribute to these associations.[37] While depression and anxiety may increase with epilepsy due to the adjustment to disability that is associated with an epilepsy diagnosis, increased depression and anxiety may also be secondary to changes in brain function common to both $\mathrm{MH}$ and epilepsy. For example, there are common neuropathological mechanisms identified in experimental epilepsy models, such as monaminergic associations with Attention Deficits Disorder (ADHD; impulsivity/disinhibition) and depressive behaviors noted in chronic epilepsy models.[38] Depression and impulsivity/disinhibition frequently co-occur, both in the general population and after TBI, with some suggestion that behavioral symptoms may be as defining of depression as dysregulated mood symptoms.[39,40] Depression may also contribute to the develop of behavioral issues,[41] emphasizing the need to identify risk factors for post-TBI depression as well and suggesting that, if PTE is associated with greater depression, it may also be a significant risk factor for behavioral issues after TBI.

Other common mechanisms include the effects of stress on seizure thresholds. Some literature suggests that stress is one of the most common self-reported precipitants of seizures among individuals in the general population with epilepsy.[42] In experimental studies, corticotropin releasing hormone $(\mathrm{CRH})$ and corticosterone elevations are shown to facilitate seizure susceptibility, presumably by altering inhibitory tone within GABAergic receptors,[43] and elevated CRH is a risk factor for post-traumatic stress disorder.[44] Preliminary clinical evidence from our laboratory indicates that elevations in serum cortisol persist beyond the first week post-TBI. Together, this evidence suggests that the stress response associated with TBI 
PTE and mental health after TBI

may contribute to $\mathrm{MH}$ symptoms, epileptogenesis, and the development of post-traumatic seizures, further facilitating a biological and reciprocal relationship between epilepsy and MH.

\subsection{Limitations}

Despite the neurobiological and clinical support for relationships between epilepsy and MH disorders, including the context of TBI, our study cannot evaluate causal relationships in our current TBI sample. As a result of the timing of adding and removing variables from the TBIMS-NDB, the present study data are cross-sectional. We were, therefore, unable to look at changes over time. Prospective, longitudinal studies collecting both seizure data (occurrence, number, timing, severity, treatment) and $\mathrm{MH}$ data (occurrence, timing, severity, treatment) are warranted to reveal the causal and reciprocal relationships that our data begin to suggest.

Data collection in this study relied primarily on self or proxy report, which is limited by poor recall, recall bias, and poor self-awareness, and the reliability and validity of self-reported health history cannot often be determined. Misclassification of PTE may have also occurred due to psychogenic seizure reporting or failing to report absence seizures, which may go unnoticed by a participant. Confirming the PTE diagnosis in medical charts was not feasible. However, despite these limitations, self-report is a common practice in studies on seizures/epilepsy.[23] Though early, immediate, and late seizures during hospitalization have a significant impact on later PTE, we were unable to make these temporal distinctions in our measure of acute seizure, which captured any seizure during the acute hospitalization stay. This temporal characterization is important for future studies. Finally, we have no information regarding previous or current medical or behavioral therapy for seizures, mental health conditions, or other comorbid conditions, all of which may have an effect on mental health outcomes. 
PTE and mental health after TBI

\section{Conclusions}

In summary, our data suggest that PTE is associated with MH outcomes 2 years after TBI. Given the literature, it is possible that psychological adjustment factors associated with PTE and/or shared biomolecular pathways may contribute to depression and anxiety in this population. Understanding the shared, and perhaps reciprocal, biological and psychological factors contributing to and resulting from both PTE and MH outcomes post-TBI is critical for treatment planning and clinical management. Further work should focus on temporal and reciprocal relationships between PTE and MH. Also, future work should consider if/how adequate PTE treatment influences MH risk, and conversely, how early identification and treatment of MH disorders may influence PTE risk over time. 
PTE and mental health after TBI

\section{Acknowledgments}

This work was supported by the National Institute on Disability, Independent Living, and Rehabilitation Research [Grants numbers 90DP0041 (SBJ, AKW, ACR, PMA), 90DP0038 (JWK), 90DP0036 (FH), 90DP0033 (WCW), 90DP0039 (RDZ)]; the Polytrauma Rehabilitation Center Traumatic Brain Injury Model System (MJP). The Polytrauma Rehabilitation Center TBI Model System is a funded collaboration between the Department of Veterans Affairs (VA) and NIDILRR. NIDILRR is a Center within the Administration for Community Living (ACL), U.S. Department of Health and Human Services (HHS). The contents of this manuscript do not necessarily represent the policy of the VA, NIDILRR, ACL, or HHS, and endorsement of this content by the Federal Government should not be assumed.

\section{Disclosure of Conflicts of Interest}

None of the authors has any conflict of interest to disclose.

\section{Ethical Publication Statement}

We confirm that we have read the Journal's position on issues involved in ethical publication and affirm that this report is consistent with those guidelines. 
PTE and mental health after TBI

\section{References}

[1] Walker WC, Ketchum JS, Marwitz JH, Kolakowsky-Hayner SA, McClish DK, Bushnik T. Global Outcome and Late Seizures After Penetrating Versus Closed Traumatic Brain Injury: A NIDRR TBI Model Systems Study. J Head Trauma Rehabil 2015;30:231-40. doi:10.1097/HTR.0000000000000127.

[2] Practice parameter: antiepileptic drug treatment of posttraumatic seizures. Brain Injury Special Interest Group of the American Academy of Physical Medicine and Rehabilitation. Arch Phys Med Rehabil 1998;79:594-7.

[3] Fisher RS, Acevedo C, Arzimanoglou A, Bogacz A, Cross JH, Elger CE, et al. ILAE official report: a practical clinical definition of epilepsy. Epilepsia 2014;55:475-82. doi:10.1111/epi.12550.

[4] Hunt RF, Boychuk JA, Smith BN. Neural circuit mechanisms of post-traumatic epilepsy. Front Cell Neurosci 2013;7:89. doi:10.3389/fncel.2013.00089.

[5] Ritter AC, Wagner AK, Fabio A, Pugh MJ, Walker WC, Szaflarski JP, et al. Incidence and risk factors of posttraumatic seizures following traumatic brain injury: A Traumatic Brain Injury Model Systems Study. Epilepsia 2016. doi:10.1111/epi.13582.

[6] Nevalainen O, Simola M, Ansakorpi H, Raitanen J, Artama M, Isojärvi J, et al. Epilepsy, excess deaths and years of life lost from external causes. Eur J Epidemiol 2016;31:445-53. doi:10.1007/s10654-015-0095-5.

[7] Harrison-Felix C, Pretz C, Hammond FM, Cuthbert JP, Bell J, Corrigan J, et al. Life Expectancy after Inpatient Rehabilitation for Traumatic Brain Injury in the United States. J Neurotrauma 2015;32:1893-901. doi:10.1089/neu.2014.3353.

[8] Beghi E. Addressing the burden of epilepsy: Many unmet needs. Pharmacol Res 2016;107:79-84. doi:10.1016/j.phrs.2016.03.003.

[9] Bushnik T, Englander J, Wright J, Kolakowsky-Hayner SA. Traumatic brain injury with and without late posttraumatic seizures: what are the impacts in the post-acute phase: a NIDRR Traumatic Brain Injury Model Systems study. J Head Trauma Rehabil 2012;27:E36-44. doi:10.1097/HTR.0b013e318273375c.

[10] Selassie AW, Wilson DA, Martz GU, Smith GG, Wagner JL, Wannamaker BB. Epilepsy beyond seizure: a population-based study of comorbidities. Epilepsy Res 2014;108:305-15. doi:10.1016/j.eplepsyres.2013.12.002.

[11] Sillanpää M, Besag F, Aldenkamp A, Caplan R, Dunn DW, Gobbi G. Psychiatric and Behavioural Disorders in Children with Epilepsy (ILAE Task Force Report): Epidemiology of psychiatric/behavioural disorder in children with epilepsy. Epileptic Disord Int Epilepsy J Videotape 2016. doi:10.1684/epd.2016.0810.

[12] Specht U, Coban I, Bien CG, May TW. Risk factors for early disability pension in patients with epilepsy and vocational difficulties - Data from a specialized rehabilitation unit. Epilepsy Behav EB 2015;51:243-8. doi:10.1016/j.yebeh.2015.07.037.

[13] Osman A, Seri S, Cavanna AE. Clinical characteristics of patients with epilepsy in a specialist neuropsychiatry service. Epilepsy Behav EB 2016;58:44-7. doi:10.1016/j.yebeh.2016.02.033.

[14] Selassie AW, Cao Y, Church EC, Saunders LL, Krause J. Accelerated Death Rate in Population-Based Cohort of Persons With Traumatic Brain Injury. J Head Trauma Rehabil 2013. doi:10.1097/HTR.0b013e3182976ad3. 
[15] Bombardier CH, Fann JR, Temkin NR, Esselman PC, Barber J, Dikmen SS. Rates of major depressive disorder and clinical outcomes following traumatic brain injury. JAMA J Am Med Assoc 2010;303:1938-45. doi:10.1001/jama.2010.599.

[16] Hart T, Brenner L, Clark AN, Bogner JA, Novack TA, Chervoneva I, et al. Major and minor depression after traumatic brain injury. Arch Phys Med Rehabil 2011;92:1211-9. doi:10.1016/j.apmr.2011.03.005.

[17] Hart T, Hoffman JM, Pretz C, Kennedy R, Clark AN, Brenner LA. A longitudinal study of major and minor depression following traumatic brain injury. Arch Phys Med Rehabil 2012;93:1343-9. doi:10.1016/j.apmr.2012.03.036.

[18] Fisher LB, Pedrelli P, Iverson GL, Bergquist TF, Bombardier CH, Hammond FM, et al. Prevalence of suicidal behaviour following traumatic brain injury: Longitudinal follow-up data from the NIDRR Traumatic Brain Injury Model Systems. Brain Inj 2016;30:1311-8. doi:10.1080/02699052.2016.1195517.

[19] Hart T, Fann JR, Chervoneva I, Juengst SB, Rosenthal JA, Krellman JW, et al. Prevalence, Risk Factors, and Correlates of Anxiety at 1 Year After Moderate to Severe Traumatic Brain Injury. Arch Phys Med Rehabil 2015. doi:10.1016/j.apmr.2015.08.436.

[20] Ritter AC, Wagner AK, Szaflarski JP, Brooks MM, Zafonte RD, Pugh MJV, et al. Prognostic models for predicting posttraumatic seizures during acute hospitalization, and at 1 and 2 years following traumatic brain injury. Epilepsia 2016. doi:10.1111/epi.13470.

[21] Corrigan JD, Cuthbert JP, Harrison-Felix C, Whiteneck GG, Bell JM, Miller AC, et al. US Population Estimates of Health and Social Outcomes 5 Years After Rehabilitation for Traumatic Brain Injury. J Head Trauma Rehabil 2014. doi:10.1097/HTR.0000000000000020.

[22] Perrin PB, Niemeier JP, Mougeot J-L, Vannoy CH, Hirsch MA, Watts JA, et al. Measures of injury severity and prediction of acute traumatic brain injury outcomes. J Head Trauma Rehabil 2015;30:136-42. doi:10.1097/HTR.0000000000000026.

[23] Fisher RS, Blum DE, DiVentura B, Vannest J, Hixson JD, Moss R, et al. Seizure diaries for clinical research and practice: limitations and future prospects. Epilepsy Behav EB 2012;24:304-10. doi:10.1016/j.yebeh.2012.04.128.

[24] Bogner JA, Whiteneck GG, MacDonald J, Juengst SB, Brown AW, Philippus AM, et al. Test-Retest Reliability of Traumatic Brain Injury Outcome Measures: A Traumatic Brain Injury Model Systems Study. J Head Trauma Rehabil 2017. doi:10.1097/HTR.0000000000000291.

[25] Fann JR, Bombardier CH, Dikmen S, Esselman P, Warms CA, Pelzer E, et al. Validity of the Patient Health Questionnaire-9 in assessing depression following traumatic brain injury. J Head Trauma Rehabil 2005;20:501-11.

[26] Kroenke K, Spitzer RL, Williams JBW, Monahan PO, Löwe B. Anxiety disorders in primary care: prevalence, impairment, comorbidity, and detection. Ann Intern Med 2007;146:317-25.

[27] Hesdorffer DC, Ishihara L, Mynepalli L, Webb DJ, Weil J, Hauser WA. Epilepsy, suicidality, and psychiatric disorders: a bidirectional association. Ann Neurol 2012;72:18491. doi:10.1002/ana.23601.

[28] Hauser WA, Annegers JF, Kurland LT. Prevalence of Epilepsy in Rochester, Minnesota: 1940-1980. Epilepsia 1991;32:429-45. doi:10.1111/j.1528-1157.1991.tb04675.x. 
[29] Hammond FM, Barrett RS, Shea T, Seel RT, McAlister TW, Kaelin D, et al. Psychotropic Medication Use During Inpatient Rehabilitation for Traumatic Brain Injury. Arch Phys Med Rehabil 2015;96:S256-253.e14. doi:10.1016/j.apmr.2015.01.025.

[30] Kumlien E, Lundberg PO. Seizure risk associated with neuroactive drugs: data from the WHO adverse drug reactions database. Seizure 2010;19:69-73. doi:10.1016/j.seizure.2009.11.005.

[31] Nickels JL, Schneider WN, Dombovy ML, Wong TM. Clinical use of amantadine in brain injury rehabilitation. Brain Inj 1994;8:709-18.

[32] Alper K, Schwartz KA, Kolts RL, Khan A. Seizure incidence in psychopharmacological clinical trials: an analysis of Food and Drug Administration (FDA) summary basis of approval reports. Biol Psychiatry 2007;62:345-354.

[33] Coupland C, Dhiman P, Morriss R, Arthur A, Barton G, Hippisley-Cox J. Antidepressant use and risk of adverse outcomes in older people: population based cohort study. BMJ 2011;343:d4551.

[34] Vermoesen K, Massie A, Smolders I, Clinckers R. The antidepressants citalopram and reboxetine reduce seizure frequency in rats with chronic epilepsy. Epilepsia 2012;53:870-8. doi:10.1111/j.1528-1167.2012.03436.x.

[35] Fann JR, Hart T, Schomer KG. Treatment for depression after traumatic brain injury: a systematic review. J Neurotrauma 2009;26:2383-402. doi:10.1089/neu.2009.1091.

[36] Barker-Collo S, Starkey N, Theadom A. Treatment for depression following mild traumatic brain injury in adults: A meta-analysis. Brain Inj BI 2013;27:1124-33. doi:10.3109/02699052.2013.801513.

[37] Valente KDR, Busatto Filho G. Depression and temporal lobe epilepsy represent an epiphenomenon sharing similar neural networks: clinical and brain structural evidences. Arq Neuropsiquiatr 2013;71:183-90.

[38] Pineda E, Jentsch JD, Shin D, Griesbach G, Sankar R, Mazarati A. Behavioral impairments in rats with chronic epilepsy suggest comorbidity between epilepsy and attention deficit/hyperactivity disorder. Epilepsy Behav EB 2014;31:267-75. doi:10.1016/j.yebeh.2013.10.004.

[39] Miller MC. When Depression Doesn’t Lead With Depression. JAMA Psychiatry 2013;70:1131-1132.

[40] Myrga JM, Juengst SB, Failla MD, Conley YP, Arenth PM, Grace AA, et al. COMT and ANKK1 Genetics Interact With Depression to Influence Behavior Following Severe TBI: An Initial Assessment. Neurorehabil Neural Repair 2016;30:920-30. doi:10.1177/1545968316648409.

[41] Juengst SB, Myrga JM, Fann JR, Wagner AK. Cross-Lagged Panel Analysis of Depression and Behavioral Dysfunction in the First Year After Moderate-to-Severe Traumatic Brain Injury. J Neuropsychiatry Clin Neurosci 2017:appineuropsych16100217. doi:10.1176/appi.neuropsych.16100217.

[42] Joëls M. Stress, the hippocampus, and epilepsy. Epilepsia 2009;50:586-97. doi:10.1111/j.1528-1167.2008.01902.x.

[43] Reddy DS, Rogawski MA. Stress-induced deoxycorticosterone-derived neurosteroids modulate GABAA receptor function and seizure susceptibility. J Neurosci 2002;22:37953805. 
PTE and mental health after TBI

[44] de Kloet CS, Vermetten E, Geuze E, Lentjes EGWM, Heijnen CJ, Stalla GK, et al. Elevated plasma corticotrophin-releasing hormone levels in veterans with posttraumatic stress disorder. Prog Brain Res 2008;167:287-91. doi:10.1016/S0079-6123(07)67025-3. 
Table 1. Demographic and Clinical Characteristics by PTE Status

\begin{tabular}{|c|c|c|c|c|}
\hline Year 1 & $\begin{array}{l}\text { PTE } \\
\mathrm{n}=54\end{array}$ & $\begin{array}{c}\text { No PTE } \\
n=399\end{array}$ & $z$ or $x^{2}$ & p-value \\
\hline Age & $39.8(17.4)$ & $40.7(18.8)$ & -0.05 & .957 \\
\hline Sex (male) & $41(75.9 \%)$ & $304(76.2 \%)$ & .002 & .542 \\
\hline Race (white) & $34(63.0 \%)$ & $290(72.7 \%)$ & 6.26 & .044 \\
\hline Pre-injury mental health treatment & $18(33.3 \%)$ & $89(22.3 \%)$ & 3.17 & .088 \\
\hline Previous TBI & $4(7.4 \%)$ & $5(1.3 \%)$ & 9.95 & $.012^{ \pm}$ \\
\hline PTA (days)* & $32.8(38.8)$ & $22.7(23.1)$ & -1.54 & .123 \\
\hline Acute seizure (yes) & $1(1.9 \%)$ & $12(3.0 \%)$ & 0.22 & .641 \\
\hline FIM (discharge) ${ }^{\star}$ & $88.2(19.6)$ & $93.2(18.6)$ & -1.85 & .064 \\
\hline FIM (follow-up) & $118.3(9.7)$ & $113.2(15.7)$ & -2.47 & .013 \\
\hline Substance Abuse (follow-up) * & $27(50 \%)$ & $158(39.6 \%)$ & 1.45 & .242 \\
\hline Year 2 & $n=33$ & $n=401$ & $\mathrm{Z}$ or $\mathrm{x}^{2}$ & p-value \\
\hline Age & $37.2(15.5)$ & $40.3(19.0)$ & -0.57 & .570 \\
\hline Sex (male) & $26(78.8 \%)$ & $294(73.3 \%)$ & 0.47 & .681 \\
\hline Race (white) & $16(48.5 \%)$ & $273(68.1 \%)$ & 12.05 & .002 \\
\hline Pre-injury mental health treatment & $8(24.2 \%)$ & $67(16.7 \%)$ & 1.21 & .335 \\
\hline Previous TBI & $2(6.1 \%)$ & $10(2.5 \%)$ & 1.30 & $.244^{ \pm}$ \\
\hline PTA (days)* & $25.5(18.8)$ & $24.7(20.5)$ & -0.46 & .649 \\
\hline Acute seizure (yes) & $8(24.2 \%)$ & $26(6.5 \%)$ & 10.00 & .002 \\
\hline FIM (discharge) ${ }^{\star}$ & $90.7(19.7)$ & $92.0(18.5)$ & -0.27 & .787 \\
\hline FIM (follow-up) & $118.8(10.4)$ & $115.4(13.5)$ & -2.63 & .008 \\
\hline Substance Abuse (follow-up) * & $12(36.4 \%)$ & $161(40.1 \%)$ & 0.20 & .712 \\
\hline \multicolumn{5}{|c|}{$\begin{array}{l}\text { Note. PTE=Post-traumatic Epilepsy; TBI=Traumatic brain injury; PTA=Post-traumatic amnesia. Race: white, black, other } \\
\text { *Year } 1 \text { ( } n=453) \text { : FIM discharge } n=442 \text {, PTA year } 1 \mathrm{n}=443 \text {, Previous TBI } n=439 \text {; Substance abuse } n=436 ; \\
\text { Year } 2(n=434) \text { : FIM discharge } n=423 \text {, PTA year } 1 \mathrm{n}=417 \text {, Previous TBI } n=439 \text {; Substance abuse } n=420 \\
\text { 'Fisher's Exact Test }\end{array}$} \\
\hline
\end{tabular}


Table 2. Depression and Anxiety Differences by PTE Status

\begin{tabular}{|c|c|c|c|c|}
\hline Year 1 & $\begin{array}{l}\text { PTE } \\
n=54\end{array}$ & $\begin{array}{c}\text { No PTE } \\
n=399\end{array}$ & $\mathrm{Z}$ or $\mathrm{X}^{2}$ & p-value \\
\hline Depressive Symptoms & $6.8(7.1)$ & $5.4(5.9)$ & -1.43 & .153 \\
\hline Depression Status + & $14(25.9 \%)$ & $84(21.1 \%)$ & 0.65 & .259 \\
\hline Anxiety Symptoms & $4.8(6.0)$ & $3.9(5.0)$ & -0.73 & .462 \\
\hline \multirow{3}{*}{$\begin{array}{l}\text { Anxiety Status + } \\
\text { Comorbid Depression } \\
\text { and Anxiety + } \\
\text { Year } 2\end{array}$} & $14(25.9 \%)$ & $81(20.3 \%)$ & 0.91 & .373 \\
\hline & $12(22.2 \%)$ & $58(14.6 \%)$ & 2.13 & .160 \\
\hline & $n=33$ & $n=401$ & $\mathrm{Z}$ or $\mathrm{X}^{2}$ & p-value \\
\hline Depressive Symptoms & $9.1(6.9)$ & $5.4(5.8)$ & -3.21 & .001 \\
\hline Depression Status + & $14(42.4 \%)$ & $86(21.5 \%)$ & 7.52 & .008 \\
\hline Anxiety Symptoms & $7.6(6.7)$ & $4.1(5.0)$ & -3.27 & .001 \\
\hline \multirow{2}{*}{$\begin{array}{l}\text { Anxiety Status + } \\
\text { Comorbid Depression } \\
\text { and Anxiety + }\end{array}$} & $14(42.2 \%)$ & $64(16.0 \%)$ & 14.49 & .001 \\
\hline & $11(33.3 \%)$ & $50(12.5 \%)$ & 10.93 & .003 \\
\hline $\begin{array}{l}\text { Note. PTE=Post-traumatic Ep } \\
\text { Depressive symptoms measu } \\
\text { measured by total score of } \mathrm{Ge}\end{array}$ & 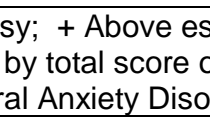 & $\begin{array}{l}\text { ent } \mathrm{H} \\
7 .\end{array}$ & & $\begin{array}{l}\text { oms; } \\
\text { ptoms }\end{array}$ \\
\hline
\end{tabular}


Table 3. Contribution of Seizures in Year 2 to Anxiety Status $(n=434)$

\begin{tabular}{|c|c|c|c|c|}
\hline & \multicolumn{2}{|c|}{ Base Model } & \multicolumn{2}{|c|}{ Base Model + Seizures } \\
\hline & OR & $p$ & OR & $p$ \\
\hline Age & & 149 & & .177 \\
\hline $31-60$ & 1.27 & .382 & 1.28 & .382 \\
\hline $61+$ & 0.51 & 159 & 0.53 & .189 \\
\hline Sex & 0.60 & .078 & 0.58 & .058 \\
\hline Race & & .034 & & .097 \\
\hline Black & 2.26 & .013 & 1.95 & .048 \\
\hline Other & 1.61 & 158 & 1.62 & 161 \\
\hline $\begin{array}{l}\text { Previous Mental } \\
\text { Health Treatment }\end{array}$ & 1.41 & .287 & 1.33 & .377 \\
\hline Seizures in Year 2 & & & 3.34 & .002 \\
\hline
\end{tabular}


Table 4. Contribution of PTE in Year 2 to Comorbid Depression and Anxiety $(n=408)$

\begin{tabular}{lcccccc}
\hline & & \multicolumn{3}{c}{$\begin{array}{c}\text { Base + Follow-up } \\
\text { Model }\end{array}$} & \multicolumn{3}{c}{$\begin{array}{c}\text { Base + Follow-up + } \\
\text { Seizures Model }\end{array}$} \\
\cline { 2 - 7 } & OR & $p$ & OR & $p$ & OR & $p$ \\
\hline Age & & .128 & & .127 & & .155 \\
$\quad 31-60$ & 1.53 & .184 & 1.54 & .177 & 1.56 & .169 \\
$61+$ & 0.55 & .298 & 0.56 & .315 & 0.61 & .401 \\
Sex & 0.77 & .439 & 0.732 & .373 & 0.69 & .302 \\
Race & & .073 & & .089 & & .130 \\
$\quad$ Black & 2.08 & .053 & 1.98 & .074 & 1.79 & .134 \\
$\quad$ Other & 1.93 & .081 & 1.95 & .079 & 1.94 & .082 \\
Previous Mental & 0.98 & .959 & 0.94 & .865 & 0.88 & .748 \\
Health Treatment & & & & & & \\
Substance Abuse & & & 1.25 & .470 & 1.32 & .376 \\
FIM (discharge) & & & 0.99 & .385 & 0.99 & .419 \\
PTE in Year 2 & & & & & $\mathbf{2 . 7 1}$ & .049
\end{tabular}

Referents: Age=16-30 years old; Sex=Male; Race=White; Absence of previous mental health treatment, substance abuse, seizures 